\title{
Las voluntades anticipadas en pacientes con esquizofrenia: un instrumento para potenciar la autonomía.
}

Advance Directives on Patients with Schizophrenia: A Tool for Maximize Autonomy.

\author{
Sergio Ramos Pozón a , Begoña Román Maestre a \\ ${ }^{a}$ Universidad de Barcelona. Facultad de Filosofía, Barcelona, España. \\ Correspondencia: Sergio Ramos Pozón (sergioramos@ub.edu)
}

Recibido: 18/02/2013; aceptado con modificaciones: 24/06/2013

\begin{abstract}
RESUMEN: En este artículo pretendemos analizar la cuestión referente al documento de voluntades anticipadas (DVA), aunque no el vinculado con el final de la vida, sino durante la vida y en un contexto psiquiátrico como es el caso de la esquizofrenia. Para ello, en primer lugar esbozamos cuáles son las características básicas del DVA. En segundo lugar, y dado que lo centraremos en la enfermedad mental, comparamos el DVA con el documento de voluntades anticipadas psiquiátricas (DVAP) viendo son sus parecidos y diferencias. También, desarrollamos cuál es el contenido y la utilidad del DVAP. Por último, realizamos algunas críticas a ambos documentos.

PALABRAS CLAVE: voluntades anticipadas, esquizofrenia y bioética.
\end{abstract}

ABSTRACT: In this paper we pretend to analyze the question referent to advance directives, although not in the end of life, but both during the life and in psychiatric context as schizophrenia First, we describe what the basic features of the advance directives are. Second, as we wish to focus in the mental illness, we compare the advance directives with advance directives in psychiatry and we expose the differences and similarities. Also, we develop which is the content and utility of advance directives. Finally, we make a critical assessment of the advance directives.

KEY WORDS: advance directives, schizophrenia, bioethics.

Agradecimientos:

Queremos agradecer al Dr. Josep Ramos, Coordinador General de los Servicios Asistenciales de Sant Joan de Déu de Salud Mental, por las significativas aportaciones críticas a este documento.

\section{1.- ¿Qué es el documento de voluntades anticipadas?}

La primera vez que se realizó una normativa jurídica que amparaba el respeto por una decisión autónoma expresada con anterioridad a una situación clínica incapacitante, fue en 1996 gracias al Convenio para la protección de los Derechos Humanos y la dignidad del ser humano con respecto a las aplicaciones de la Biología y la Medicina. En el artículo 9 se dice que "serán tomados en consideración los deseos expresados anteriormente con respecto a una intervención médica por un paciente que, en el momento de la intervención, no se encuentre en situación de expresar su voluntad". En nuestro país, sin embargo, hubo que esperar hasta el año 2000 para que en Cataluña, gracias a la Ley 21/2000, sobre los derechos de información relativos a la Salud, la autonomía del paciente y la documentación, 
se reconociese este derecho mediante el documento de voluntades anticipadas. Posteriormente, a nivel estatal entró en vigor la Ley 41/2002, de 14 de noviembre, básica reguladora de la autonomía del paciente y de derechos y obligaciones en materia de información y documentación clínica, y después muchas comunidades autónomas elaboraron su propia legislación. En Cataluña, tanto el Comité de Bioètica de Catalunya (1) como el Grupo de Opinión del Observatori de Bioètica $i$ Dret (2) han redactado documentos en los que se explican el contenido, utilidad, situaciones clínicas asociadas al proceso, fundamentos éticos, etc. Pese a la difusión de la existencia del documento de voluntades anticipadas y de su reconocimiento jurídico, es muy poco conocido tanto por los pacientes como por los profesionales ${ }^{1}$ (3-4).

La finalidad de este documento es dejar constancia de la expresión de la voluntad y preferencias que emite una persona autónoma para que, en el momento de carecer de competencia suficiente, se tenga en cuenta su decisión. Se trata, por tanto, de respetar y promocionar la autonomía del paciente en momentos de vulnerabilidad. Marc Antoni Broggi (5) opina que es más importante la confianza mutua entre médico y paciente que el documento en sí, el cual puede convertirse en un contrato despersonalizado. En efecto, lo verdaderamente importante es el proceso dialógico en el que equipo médico y paciente (y muchas veces también familia y/o tutor o representante) llegan a acuerdos consensuados sobre qué hacer y qué no hacer en el momento de la situación incapacitante. Este acuerdo se basa en el respeto y la confianza mutua, por lo tanto, es una exigencia ética. Por el contrario, si la única finalidad es conseguir que el paciente redacte el documento para eximir al equipo médico de responsabilidades, entonces se reduce a un procedimiento meramente jurídico y de una exigencia administrativa.

Tanto en el procedimiento ético como en el administrativo conseguimos una relación asistencial más trasparente al conocer los deseos y preferencias del paciente. Su conocimiento ayuda a que la familia no tenga que decidir por el paciente y da garantías de que habrá respeto por la voluntad de éste.

Ahora bien, es necesario que la decisión que se tome no sea "ciega", basada únicamente en deseos y preferencias del paciente, sino fundamentada también en el conocimiento científico, en una valoración de la situación y en una indicación médica por parte de los profesionales, estableciéndose así un proceso reflexivo mutuo. Y es que no es posible que el paciente escoja libremente cualquier opción, pues su voluntad, si quiere que sea respetada, ha de ser acorde con la "lex artis"

El estudio de Molina J, et al. (3) realiza una encuesta a 155 pacientes de un hospital público de la Comunidad de Madrid sobre el conocimiento del documento de voluntades anticipadas. Sólo 7 personas (4,5\%) tenían constancia de su existencia y únicamente 1 lo tenía realizado. Otro problema añadido es que, pese al conocimiento de los profesionales de la existencia del documento, saben poco de la normativa, del contenido y de su registro (Champer A., et al. (4)). 
y no contrarias al ordenamiento jurídico. Además, la decisión se respetará si corresponde el supuesto que dictaminó con la situación actual. Esta exigencia jurídica lleva implícita la idea de que las voluntades, antes de quedar plasmadas en el documento, han de ser evaluadas para analizar qué se está solicitando, cómo y por qué, e incluso podría llegar a ser aconsejable algún tipo de modificación para que sean acordes con las buenas praxis médicas.

Núria Terribas (6) sostiene que solicitar que las decisiones sean acordes a la "lex artis" puede confundirse con aquello que el médico considere qué es buena praxis, de manera que es preferible entender por "buena praxis" lo consensuado en protocolos o guías de especialidad. El hecho de que no tengan que ser contrarias al ordenamiento jurídico se debe al deseo de evitar la inclusión de petición de eutanasia. Por último, Terribas cree que es muy difícil que coincida la voluntad del paciente con la exacta situación clínica. Por esta razón, la figura del representante va a tener un papel crucial a la hora de interpretar los deseos del paciente.

La Ley 41/2002, de 14 de noviembre básica reguladora de la autonomía del paciente y de derechos y obligaciones en materia de información y documentación clínica, art. 11.1, contempla la posibilidad de que el otorgante designe un representante para que haga de interlocutor válido con el equipo médico y así velar por el cumplimiento de las instrucciones previas y respetar su autonomía. El conocimiento de sus deseos y preferencias puede evitar malentendidos o disputas sobre qué decisiones médicas llevar a cabo. La persona escogida como representante ha de ser de confianza para el paciente, puesto que es muy importante que conozca cuál es su voluntad. Se aconseja dejar constancia de más de un representante, a modo de sustituto, aunque especificando quién es el que tiene prioridad para llevar la tarea. En principio, no puede contradecir aquello que la persona ha dejado constancia en el documento, de manera que tiene que actuar según el criterio y las instrucciones expresadas. No obstante, cabe la posibilidad de que proceda en situaciones no especificadas como, por ejemplo, en contextos en los que sea preciso un consentimiento informado o en decisiones que no se hayan contemplado en el documento. Es aconsejable que la familia tenga conocimiento de esta figura fiduciaria, pues será quien tomará la decisión (1). Pese a que se recomienda que no tenga ningún parentesco familiar ni que forme parte del equipo profesional, Marc Antoni Broggi (5) sostiene que podría ser nombrado como representante un sanitario (médico de familia, enfermera amiga...), aunque posteriormente quede excluido para tomar las decisiones como facultativo. Por nuestra parte, también compartimos esta opinión. Un médico de familia y/o el equipo de enfermería conocen algunos aspectos personales del paciente (cultura, convicciones, creencias...) así como su relación familiar. Por otro lado, también tienen constancia de cuál es su enfermedad, curso y pronóstico, de manera que están más capacitados para hacerse una panorámica más amplia de su situación 
clínica particular. Además, gracias a su proximidad con el paciente, el otorgante suele depositar la confianza en alguna de estas figuras, puesto que son ellos los que se dedican a "cuidarle".

Aunque el paciente haya dejado constancia de un representante para que lleve a cabo su voluntad, sin unas instrucciones y guías precisas sobre qué está solicitando se corre el riesgo de que el representante no sepa determinar con claridad qué deseos exactamente determinan su voluntad. En este sentido, Pablo Simón e Inés Barrio (7), apelando a Emanuel et al. (8), señalan algunos puntos que pueden contribuir a una mayor comprensión respecto a qué instrucciones previas anotar y cómo ejecutarlas. Entre ellas, destacamos las siguientes:

1. Invitar al paciente a que explique qué papel espera de su representante.

2. Solicitar al representante que aclare con el paciente aquellos valores y criterios que deberían de usarse en el momento de incapacidad.

3. Preguntar al paciente si ha tenido anteriores experiencias de la enfermedad, o bien si tiene constancia de otras personas con la misma situación, e invitarle a que comente su opinión y sentimientos al respecto.

4. Sugerir al otorgante que manifieste lo que desearía que se hiciera en tales contextos clínicos.

5. Ayudarle a que articule sus propios deseos, valores y preferencias en relación a esas situaciones.

6. Animarle para que deje constancia por escrito de alguno de estos casos.

Todas estas sugerencias, así como el análisis del documento en sí, suelen estar enfocadas a situaciones incapacitantes, a saber; coma o estado vegetativo, enfermedad irreversible en fase avanzada o terminal, demencias severas... También es frecuente el rechazo de medidas de soporte vital (diálisis o ventilación mecánica, entre otras, reanimación cardipulmonar...) En definitiva, suele estar vinculado con la toma de decisiones al final de la vida. Ahora bien, es posible realizar este procedimiento no con vistas al final de la vida, sino a situaciones incapacitantes durante la vida. Este puede ser el caso de las voluntades anticipadas en el ámbito psiquiátrico. Aunque comparten muchas de las ideas principales con el documento de voluntades anticipadas tradicional, tiene un contenido y una utilidad totalmente distintos.

2.- Diferencias entre Documento de Voluntades Anticipadas y Documento de Voluntades Anticipadas en Psiquiatría

El Documento de Voluntades Anticipadas (DVA) y el Documento de 
ORIGINALES Y REVISIONES

Voluntades Anticipadas en Psiquiatría (DVAP) comparten la idea general de que el objetivo es dejar constancia de la voluntad del paciente para cuando no pueda expresarla de manera competente, que han de elaborarse mientras la persona es competente, y que sus deseos han de ser claramente expresados y acordes a la normativa jurídica y a la "lex artis"; sin embargo, hay una serie de diferencias notorias entre ambos documentos. Robert Miller (9) señala, entre otras, las siguientes:

1. En el DVA los pacientes son, en un tiempo relevante, totalmente incapaces de expresar sus deseos, mientras que en el ámbito psiquiátrico rara vez son incapaces o incluso pueden ser reacios a expresar sus decisiones.

2. Los DVA, cuando son llevados al caso clínico, suelen tener una connotación meramente negativa (rechazo de un tratamiento); en los DVAP, al menos en teoría, cabe la posibilidad de que se expresen deseos negativos (rechazos) pero también positivos (elección de tratamiento, preferencias de hospitalización, etc.).

3. Las decisiones que toman los enfermos terminales no afectan al pronóstico de la enfermedad, ya que sustancialmente se refieren a que su muerte se produzca antes o después, o al grado de sufrimiento producido durante el proceso. En pacientes psiquiátricos la elección de un tratamiento incluye la hospitalización para proteger al paciente y a otras personas, y para activar un tratamiento que tiene resultados favorables.

Otra gran diferencia es que en pacientes con enfermedad mental el nivel de conciencia se mantiene, y no como sucede con un paciente en coma, aunque la persona no esté capacitada de facto en ese momento. Y aquí puede darse la paradoja de que el paciente rechace, debido a estados delirantes, una opción que había elegido previamente (10).

Por nuestra parte, añadimos la distinción de que en el DVAP tiene más sentido la revisibilidad de la voluntad expresada. Al comienzo del abordaje terapéutico es frecuente que se pruebe con distintos tipos de fármacos y de dosis para ver qué tratamiento es el que mejor se adapta a las características del paciente. Esto es más probable en los primeros ingresos. Y dado que los antipsicóticos suelen tener reacciones adversas que no todos los pacientes toleran igual, es posible que el paciente rechace específicamente algún tipo de fármaco. El conocimiento de éstos y de cómo afecta puede conllevar a la modificación del documento. De manera que nuevamente se tiene que realizar una valoración de la competencia para verificar que se trata de una decisión autónoma y competente. Con la revisibilidad del documento se da más garantías de que es la voluntad actual del paciente y con ello un mejor respeto y atención a la autonomía del paciente.

Pese a que en principio en un DVA se puede rechazar cualquier tipo de 
tratamiento, en el ámbito psiquiátrico el rechazo a toda medida terapéutica no está tan clara. Pensamos que no es sensato que un paciente con esquizofrenia rechace todo tipo de medicación psiquiátrica durante su ingreso ${ }^{2}$ (11-13), pues es ésta la que va a posibilitar la eliminación de la sintomatología positiva (alucinaciones, delirios...). Un brote psicótico puede, aunque no necesariamente, conllevar conductas auto y heteroagresivas, de modo que el no recibir tratamiento farmacológico puede poner en peligro la vida del paciente y/o la de otras personas. Además, el rechazo de una medida terapéutica, que tiene unos resultados positivos y que son la base del actual tratamiento psiquiátrico, puede indicar algún tipo de incompetencia por parte del paciente debido a la falta de información sobre su beneficio, a la sobreestimación de los efectos secundarios, o a decisiones fundamentadas en delirios, etc.

\section{3.- Contenido y utilidad del DVAP}

Como suele suceder con el DVA, el DVAP normalmente se enfoca a instrucciones previas relacionadas con el tratamiento. Los pacientes pueden rechazar o preferir específicamente algún tipo de fármaco antipsicótico ${ }^{3}$ (14-15).

Al indicar cuáles son las preferencias no se hace alusión únicamente a lo relacionado con el tratamiento, sino también a cuestiones vinculadas con la hospitalización. Es posible que los pacientes psiquiátricos, en momentos de crisis en los que se cuestione la posibilidad de un ingreso hospitalario, prefieran alternativas a la hospitalización convencional. Los motivos que aluden son el poco cuidado cualificado y el poco respeto de los profesionales. Como alternativas suelen mencionar el deseo de permanecer en casa y descansar mientras se produce la crisis, tener la oportunidad ser visitados por el profesional de la salud en su domicilio, etc. (15).

También es posible incluso otra serie de instrucciones en el DVAP que aluden a la hospitalización. Los pacientes no siempre toleran todas las visitas, de modo que en ocasiones pueden pedir, expresamente, que no acuda algún familiar o amigo

\footnotetext{
Este fue el caso Hargrave v. Vermont en el año 1999. Nancy Hardgrave, una mujer con esquizofrenia, había realizado su DVAP rechazando todo tipo de medicación psiquiátrica. Un análisis del caso se encuentra en Appelbaum P. (11). Aunque es posible un caso como éste, los estudios empíricos muestran que los pacientes no suelen rechazar todos los fármacos (Backlar P., et al. (12) y Swanson J., et al. (13)).

En el estudio de Wilder Ch, et al. (14) se indica, en una muestra de 123 personas con enfermedad mental grave, cuáles son los más aceptados y rechazados. Los fármacos risperidona, olanzapina, y quetiapina han sido los más aceptados $(20 \%, 17 \%$ y $15 \%$, respectivamente), mientras que el más rechazado ha sido el haloperidol (24\%). Con ello, se constata que los antipsicóticos de segunda generación son preferidos antes que los de primera generación. Los motivos que alegan los pacientes para su rechazo son las reacciones adversas, sentirse "dopados", insomnio y/o no permitirles realizar las actividades diarias (15). Otro tratamiento que suelen rechazar es la Terapia Electroconvulsiva (TEC). Srebnik, D, et al. (15) indican que de una muestra de 106 pacientes el $72 \%$ rechazaban la TEC, mientras que sólo el $16 \%$ estaban a favor.
} 
ORIGINALES Y REVISIONES

$\mathrm{y}$, al contrario, que durante el ingreso se contacte con alguien en particular. Puede darse el caso, incluso, que se otorgue a esa persona de interés el cuidado de algún familiar dependiente, de una mascota... Otra cuestión a que se suele aludir son las preferencias o rechazos sobre la dieta (15-16).

El DVAP también tiene la utilidad de tener repercusiones tanto en la recuperación de la persona como en la mejora asistencial. En efecto, la aceptación y deseo de algún tipo de fármaco tiene resultados terapéuticos positivos. Está demostrado que la libre elección del tratamiento, el conocimiento de sus contraindicaciones y la importancia de su seguimiento tienen una mejor adherencia farmacológica y una reducción del número de recidivas. Y es que la elección del tratamiento conlleva una motivación para su seguimiento y este cumplimiento terapéutico tiene unos resultados positivos para el bienestar físico y psicológico del paciente. Por otro lado, también se sabe que la no adherencia a los fármacos está asociada al tratamiento involuntario y a un peor curso clínico (13,15,17-19).

Visto desde el punto de vista de la relación asistencial, el proceso de la elaboración del DVAPestá señalando a un modelo de toma de decisiones compartidas (20) en el que se dan decisiones conjuntas entre el paciente, familia y profesionales sobre cuál es la mejor línea terapéutica y qué decisiones futuras tomar. Se trata de que el paciente exprese su voluntad pero que lo haga con el asesoramiento y recomendación del equipo médico. Esto supone que durante este proceso se ayuda a clarificar cuál es la voluntad del paciente y cuál es la mejor opción teniendo en cuenta su punto de vista, pero siempre respetando su decisión final que será la que se recoja en el DVAP. No hay que olvidar que las personas tienen el derecho a elegir y/o rechazar un tratamiento siempre y cuando sea acorde con la normativa vigente, como son la Ley 21/2000, de Cataluña, sobre los derechos de información relativos a la salud, la autonomía del paciente y de la documentación clínica y la Ley 41/2002, de 14 de noviembre básica reguladora de la autonomía del paciente y de derechos y obligaciones en materia de información y documentación clínica.

Dando la oportunidad al paciente de poder escoger libremente se contribuye al aumento de la impresión de empoderamiento. Se trata de darle más protagonismo a la persona sobre las decisiones que hacen alusión a su proyecto vital. Backlar P, et al. (12) constataron que los pacientes que habían realizado el DVAP estaban más satisfechos consigo al sentirse más protegidos por saber que, en momentos de mayor vulnerabilidad, eran ellos los que tomarían las decisiones y no el equipo médico. Saber que habrá respeto por su voluntad también tiene un componente positivo al reducir la sensación de estar recibiendo un tratamiento coercitivamente, por lo que será más fácil una colaboración durante el proceso. A su vez, si la persona percibe que su voluntad no es respetada, apreciará este hecho como una injusticia y una exclusión, que redundará en una disminución del seguimiento terapéutico (17). El uso del DVAP no reduce únicamente el "sentimiento" de coerción, sino 
también el uso de medidas coercitivas, como requerir de los servicios policiales, en ocasiones esposándolo, para la evaluación de su competencia; contención mecánica; medicación obligatoria... En efecto, si hay una mayor participación y colaboración en la relación asistencial es posible una reducción de algunas de estas medidas restrictivas. Si hay confianza entre equipo médico y paciente, los clínicos podrían ayudar a reducir esas intervenciones coercitivas e incluso del período de ingreso involuntario. Por otro lado, si hay constancia de un representante en el DVAP, éste puede consentir que se dé un cierto tratamiento, si es preciso, con el que evitar un ingreso involuntario (21). Además, hay más posibilidad de que haya un respeto por la voluntad del paciente cuando en el DVAP se ha dejado constancia de un representante al que acudir (15).

\section{4.- Críticas al documento de voluntades anticipadas}

Tanto el DVA como el DVAP constituyen una buena herramienta para la toma de decisiones; sin embargo, también conlleva problemas e inconvenientes que pueden entorpecer su aplicación y justificación.

Mark R. Tonelli (22) realizó un artículo centrado en las críticas que pueden realizarse al DVA. Entre ellas, destacamos las vinculadas con la teoría de la identidad y personalidad, los inconvenientes prácticos de su aplicación y los problemas derivados de la utilización de un representante.

Las críticas derivadas de la teoría de la identidad y personalidad ${ }^{4}$ (23) se basan en el hecho de que la persona que ha realizado el DVA y la persona a la que está destinada su contenido son diferentes. Es preciso que haya una continuidad en la identidad o personalidad respecto a los intereses y valores. $\mathrm{Y}$ en situaciones incapacitantes derivadas de una enfermedad severa o de la pérdida de funciones cognitivas, los valores de referencia que tomó la persona a la hora de elaborar el DVA ya no corresponden con los de la persona incapacitada. Éste fue el caso de Margo, una mujer con alzheimer (24). Se parte de la tesis de que la persona Margo que realizó el DVA ya no es exactamente la misma, sino que se trata de una persona demenciada que es totalmente distinta debido al deterioro neurodegenerativo de la enfermedad. Con ello, los intereses y valores entre ambas personas entran en conflicto. Ben Rich (24), apelando a Richard Dworkin, sostiene que esta contradicción puede solventarse mediante la distinción de dos tipos de intereses,

\footnotetext{
Buchanan y Brock (pp:152-189) desarrollan con más detalle esta crítica analizando varios temas, a saber: qué significa la pérdida de identidad, qué se requiere para que haya "continuidad psicológica" o cuáles son los intereses de la persona. Por motivos de espacio, no es posible reconstruir aquí todo el argumento, pero en líneas generales, para solventar las críticas a la teoría de la identidad apelan a que las teorías de la identidad han de ser comprendidas en el sentido en el que hay continuidad psicológica gradual y no concebirse como un todo o nada.
} 
ORIGINALES Y REVISIONES

a saber: experienciales e intrínsecos. Los primeros hacen alusión a aquellos tipos de intereses que una persona experimenta como agradables, pero que no todas las personas comparten. Los intereses intrínsecos son esos valores que hacen que una vida se convierta en genuinamente mejor que otra y además ésta sería peor si no se reconociesen. En la búsqueda de intereses intrínsecos se revela significativamente la identidad de la persona y no en los experienciales. Por nuestra parte, también considero que en el DVA se deja constancia de los intereses intrínsecos, en el sentido de reflejar por qué quiero yo una determinada calidad de vida y no simplemente cómo quiero vivir. Se trata de la notificación de intereses que son acordes con un proyecto de vida elegido libremente y que son los que dan sentidos a su vida.

Pablo Simón e Inés Barrio (7) sostienen que esta crítica puede ser relevante en el caso de demencias; sin embargo, en general se asume que las personas poseen una cierta identidad personal a lo largo de su vida, pues de lo contrario los actos que realizamos a diario no serían asignables a los que se realizarán en un futuro a corto plazo. Ejemplo de ello son los testamentos para legar bienes que serían inválidos por ser distintas personas.

Mark R. Tonelli (22) también critica el modo en el que el representante lleva a cabo la voluntad del paciente. Las decisiones que toman los representantes han de ser acordes con aquello que querría el paciente, pero dado la dificultad que conlleva, en la gran mayoría de casos se intenta aplicar el criterio del juicio sustitutivo. El criterio del juicio sustitutivo es que una persona competente toma en nombre de otro que es incompetente. El problema es que hay poca concordancia entre los deseos del paciente con los elegidos por el representante, pues no concuerdan exactamente al tratarse de interpretaciones sobre la voluntad del paciente, de modo que al final se convierte en un ideal y no en una realidad. Por tanto, la justificación válida de un representante no se encuentra en la aserción de que éstos significan una extensión de la autonomía del paciente, sino que se trata de la realización práctica de una decisión en nombre de un paciente incompetente.

Otra posible crítica que apela el autor estriba en la posibilidad de que aquellos pacientes que realizan el DVA hayan podido tener una información incompleta sobre los riesgos y beneficios de los tratamientos. Aunque es posible una defensa a este ataque al solicitar a los clínicos más habilidades con las que realizar mejor el proceso informativo, una información exhaustiva de los pros y contras sólo podría darse si se revisaran un máximo de 3 ó 4 tratamientos. Y es que no es posible una indagación rigurosa de todos los tratamientos que puedan tener una cierta utilidad clínica para el caso concreto. Tampoco hay métodos rigurosos que aseguren que se informa adecuadamente.

Por último, el autor sostiene que el modo en el que están redactados los DVA también puede ser objeto de crítica, por reflejar un lenguaje vago e impreciso sobre algunas situaciones médicas, a saber: condición terminal, enfermedad 
incurable, incapacidad seria... que al final suele depender de la interpretación de los profesionales. Además, si se intentan especificar con instrucciones precisas respecto a qué se quiere decir con dichos términos, puede dar lugar a la exclusión de pacientes por no corresponder con la exacta situación clínica. Los intentos de tomar instrucciones previas más resistentes a interpretaciones acaban por tener una menor probabilidad de ser aplicadas, pues si especificamos exactamente en qué contextos clínicos queremos que sean aplicadas las instrucciones previas, todos aquellos contextos que no correspondan de manera exacta no serán tenidos en cuenta a la hora de aplicar las voluntades anticipadas.

Otros autores como Pablo Simón e Inés Barrio (7) también han mostrado algunas insuficiencias. Ellos las agrupan en 4 epígrafes: 1) críticas a la fundamentación; 2) críticas a la validez; 3) críticas a la hermenéutica; y 4) críticas a la utilidad. A continuación vamos a desarrollar sólo algunas de éstas, pues la gran mayoría ya han sido tratadas anteriormente.

En cuanto a la validez hay que hacer mención a los problemas de caducidad en el que se cuestiona si los deseos del paciente aún son vigentes desde que se redactó el documento hasta el momento de su aplicación. Los autores citan algunos estudios que indican que más allá de 2 años no se puede asegurar la duración de las decisiones. Las críticas a la hermenéutica hacen alusión al hecho de que la persona, cuando acepta o rechaza una determinada línea terapéutica, lo hace en situaciones hipotéticas en la que es imposible prever todas las variables clínicas que intervienen en la situación real. Por eso, puede darse el caso de que, al aplicar el DVA a la situación real, con toda seguridad el paciente no la hubiese querido por ir en contra de sus mejores intereses. Esto se debe a que la situación real puede modificar el diagnóstico, tratamiento o pronóstico.

Pero cabe también constatar otros problemas del documento cuando se centra en el ámbito psiquiátrico. En este sentido, Richard Van Dorn et al. (25), al entrevistar a 591 profesionales (psiquiatras, psicólogos y trabajadores sociales), han constatado una serie de barreras que dificultan la aplicación del DVAP. Las más puntuadas fueron: 1) falta de acceso al DVAP por parte de los profesionales; 2) carencia de un equipo médico entrenado o con conocimientos sobre cómo ha de ser aplicado y manejado el DVAP; y 3) incomunicación entre el equipo de profesionales sobre la existencia de dicho documento. Por otro lado, Kaustubh Joshi (26) sostiene que muchas personas con enfermedad mental tienen dificultades para entablar relaciones interpersonales, lo que dificulta la posibilidad de poder tener un representante. Por otro lado, pese a que el paciente haya redactado su DVAP, no por ello todos los hospitales y clínicos han de tener conocimiento y acceso. Por esta razón es muy importante hacer una difusión tanto a los familiares y representante, como al centro de salud que suele visitar.

Por nuestra parte, podemos considerar otras dificultades. Es posible que 
ORIGINALES Y REVISIONES

algunos pacientes con esquizofrenia tengan nula conciencia de patología, de modo que difícilmente se cuestionarán la necesidad de elaborar un DVA. Por otro lado, cabe la posibilidad de que tengan dificultades para tomar decisiones autónomas, a saber: problemas de atención, déficit en el almacenamiento de la memoria, pobre procesamiento y comprensión de nueva información, insuficiencias en la función ejecutoria (idear y llevar a cabo planes), dificultades en la memoria verbal, desorganización conceptual, etc. Se han elaborado herramientas para revisar y mejorar la competencia de los pacientes a la hora elaborar un DVAP. Este es el caso del Competence Assessment Tool for Psychiatric Advance Directives (CATPAD) diseñado por Srebnik, Appelbaum y Russo (27). Esta herramienta analiza la información relevante, la apreciación de su propio significado y el razonamiento en un proceso racional de ponderación de riesgo/beneficio. Y dado que puede haber dificultades en algunas de estas áreas, se ha creado un programa informático que ayuda a educar a los pacientes sobre sus deseos, preferencias, elecciones, etc., y facilitar la creación del DVAP de manera consistente (28).

Dejando de lado la competencia necesaria para la elaboración del DVAP, cabe preguntarse si siempre es factible su aplicación en todos los contextos clínicos. Es aceptado que en el momento de una crisis aguda se ha de actuar de manera urgente para evitar conductas auto y heteroagresivas. Esta situación de urgencia puede condicionar al hecho de que se tenga que actuar sin tiempo suficiente para ver si la persona tiene un DVAP. La ley ampara que se actúe en situaciones de urgencia sin tener que recurrir al consentimiento informado, y también esto es aplicable al DVAP. Ejemplo de estas leyes son Ley General de Sanidad, el Convenio de Oviedo (1997) y la Ley 41/2002, de 14 de noviembre, entre otras.

Por último, dado que en nuestro país no hay normativa jurídica específica que respalde el derecho a las voluntades anticipadas en el ámbito psiquiátrico ${ }^{5}$, tenemos que apelar a la Ley 41/2002. En esta se reconoce el derecho a la autonomía pero solicita que sean acordes con la lex artis; sin embargo, en casos clínicos en los que no se sepa con seguridad qué decisión tomar, la interpretación sobre qué se entiende por lex artis estará en manos del profesional. Esto puede suponer la revocación de las DVAP al buscar el mayor beneficio del paciente, de modo que la interpretación del significado de lex artis puede conllevar un modelo paternalista y, por tanto, la anulación del derecho a la autonomía. En casos de incertidumbre es preferible solicitar consejo a un Comité de ética asistencial.

\footnotetext{
En este sentido, aún estamos muy atrasados en materia legislativa en relación a la salud mental. Por ejemplo, en Estados Unidos muchos estados han elaborado leyes que avalan el respeto por estos documentos. Para una mayor información sobre cuál es la situación específica de cada estado puede consultarse la página web http:// www.nrc-pad.org/, visitada el 18 de febrero de 2013.
} 
ORIGINALES Y REVISIONES

\section{5.- Conclusiones}

Por regla general, el DVA ha sido enfocado exclusivamente al proceso del final de la vida, por lo que el contenido y la utilidad clínica se han centrado en el proceso de morir. Aunque es de gran importancia ese ámbito, también es posible aplicar el documento para dejar constancia de la decisión del paciente durante el proceso de la vida en otros contextos como el socio-sanitario en el ámbito psiquiátrico.

El problema está en que a la hora de realizar y aplicarlo a la Psiquiatría es posible constatar una serie de inconvenientes. Se exige que sea elaborado por una persona competente, pero los pacientes con esquizofrenia pueden tener problemas de atención, déficits en el almacenamiento de la memoria, pobre procesamiento y comprensión de nueva información, etc., que pueden suponer incompetencia para tomar decisiones. Además, en ocasiones no tienen conciencia de patología, de modo que consideran que no necesitan la medicación porque no están enfermos, lo que supone que no se replantean los posibles ingresos debido a una recaída y con ello no valoran la necesidad de elaborar el documento. Por otro lado, cuando intentamos aplicar el DVAP a la praxis médica también tenemos dificultades. En el contexto psiquiátrico son frecuentes las descompensaciones psicóticas que requieren de una rápida intervención, lo que supone actuar sin tener tiempo suficiente para ver si se ha dejado constancia del DVAP ni de valorar si aquello que se ha notificado sea lo más positivo para la situación particular. E incluso en situaciones que no son de urgencia en las que hay que tomar decisiones, si se ha dejado constancia de un representante que lleve a cabo la voluntad del paciente mediante un juicio por representación, esto tampoco asegura que se refleje con fidelidad la decisión del paciente, de manera que se puede cuestionar la utilidad del representante y por lo tanto la del documento. Ahora bien, hemos de tener en cuenta que es posible que hayan imprevistos no contemplados en el DVAP, siendo aquí donde hay que emitir ese juicio por representación, valorar la circunstancia y juzgar qué es lo mejor para el paciente, pero siempre teniendo en cuenta su escala de valores y sus deseos previos. Y esto no significa, necesariamente, la anulación ni de la utilidad del representante ni del documento.

El tener que anticiparnos a los imprevistos hace que cobre mayor importancia el diálogo entre representante y paciente para saber cómo hemos de actuar.Además, puede darse el caso de que la persona cambie de opinión respecto al tratamiento debido a la intolerancia de los medicamentos, de la interacción con otros fármacos, que se intente con tratamientos alternativos, etc., y de todo ello el representante ha de tener constancia. Es por eso que es muy importante una revisión periódica del documento en el que se evalúe qué se pide y cómo, y esto va a suponer la valoración, nuevamente, de la competencia del paciente.

Junto con las dificultades de aplicación del documento, hay que añadir otras 
ORIGINALES Y REVISIONES

insuficiencias metodológicas, pues hemos dejado abiertas, o sin resolver, algunas cuestiones. En primer lugar, la necesidad de elaborar una normativa jurídica en el ámbito psiquiátrico o una revisión de la Ley 41/2002 que ampare el derecho de los pacientes psiquiátricos a dejar constancia del DVAP. En segundo lugar, puesto que es frecuente que en pacientes con esquizofrenia se tenga que emitir juicios por representación, se hace necesario desarrollar y especificar con más claridad en base a qué, cómo y de qué manera hemos de representar a la persona. A su vez, hay que analizar quién es el que está más capacitado para emitir con mayor precisión su voluntad.

Por último, hay que dejar claras unas cuestiones. Este documento en salud mental es especialmente importante porque el objetivo es asumir que la persona tiene una enfermedad y ayudar a proyectar su vida en un momento de incapacidad, facilitando así la toma de decisiones a quienes tienen que tomar decisiones cuando él no pueda hacerlo. Es por este motivo por el que el documento de voluntades anticipadas ha de entenderse como un instrumento para potenciar el respeto a la autonomía del paciente a lo largo de la vida, con la enfermedad, y no sólo enfocado al final de la vida.

Ahora bien, este documento no puede determinar, de manera definitoria, qué tipo de tratamiento desea, ni oponerse a cualquier medida terapéutica. $Y$ es que en una descompensación psicótica la persona no podrá rechazar cualquier medida antipsicótica y/o negar un ingreso involuntario, pues en ningún caso se pondrá en riesgo la salud de la persona como puede ser debido a algún tipo de conductas auto y heteroagresivas. Además, los DVAP pueden ser una herramienta que ayude a mejorar la consciencia de patología del paciente, ya que hace que se tenga que replantear cuestiones sobre la enfermedad y sus repercusiones; y a tomar decisiones compartidas respecto a las preferencias del paciente, así como en momentos en los que se tenga que tomar una decisión por representación debido a incompetencia. Con ello, el DVAP facilita el empoderamiento del paciente en la toma de decisiones, pues se le introduce en la toma de decisiones y se le da más protagonismo sobre las cuestiones referencias a su salud. En definitiva, la realización del DVAP conlleva una mejora en la relación asistencial, en el cumplimiento del tratamiento y por tanto en la recuperación de la persona, y contribuye a respetar el derecho a la autonomía $(14-15,17-19,21)$.

\section{BIBLIOGRAFÍA:}

(1) Comitè de Bioètica de Catalunya. Departament de Sanitat i Seguretat Social de la Generalitat de Catalunya. Consideracions sobre el document de voluntats anticipades. Barcelona: Direcció General de Recursos Sanitaris; 2002.

(2) Grupo de Opinión del Observatori de Bioètica i Dret Parc Científic de Barcelona. 
Documento sobre las voluntades anticipadas. Barcelona; 2001.

(3) Molina J, Pérez M, Herreros B, Martín M y Velasco M. Conocimiento y actitudes sobre las instrucciones previas entre los pacientes de un hospital público de la Comunidad de Madrid. Rev Clin Esp. 2011;211(9):450-454.

(4) Champer A, Caritg F y Marquet R. Conocimientos y actitudes de los profesionales de los equipos de atención primaria sobre el documento de voluntades anticipadas. Aten Primaria.2010;42(9):463-469.

(5) Broggi M.Las voluntades anticipadas. Humanitas, Humanidades médicas. 2003;1(1):6170.

(6) Terribas N. Las voluntades anticipadas y su utilización en la toma de decisiones. Vectores de Investigación. 2012;5(5):17-26.

(7) Simón Py Barrio I. ¿Quién decidirá por mí? Ética de las decisiones clínicas en pacientes incapaces. Madrid: Triacastela; 2004.

(8) Emmanuel L, Gunten C y Ferris F. Advance Care Planning. Arch Fam Med. 2000;9:11811187.

(9) Miller R. Advance Directives for Psychiatric Treatment. A view from the Trenches. Psychology, Public Policy, and Law. 1998;4(3):728-745.

(10) Gorrotxategi M y Romero J. Voluntades psiquiátricas: Una herramienta terapéutica. Norte de salud mental. 2012;10(42):11-17.

(11) Appelbaum P. Psychiatric Advance Directives and the Treatment of Committed Patients. Psychiatric Services. 2004;55(7):751-753.

(12) Backlar P, McFarland B, Swanson J y Mahler J. Consumer, Provider, and Informal Caragiver Opinions on Psychiatric Advance Directives. Administration and Policy in Mental Health, 2001;28(6):427-441.

(13) Swanson J, Swartz M, Elgogen E, Van Dorn R, Ferron J, Wagner H, McCauley B y Kim M. Facilitated Psychiatric Advance Directives: A Randomized Trial of an Intervention to Foster Advance Treatment Planning Among Persons with Severe Mental Illness. Am J Psychiatry. 2006;163:1943-1951.

(14) Wilder Ch, Elbogen E, Moser L, Swanson J y Swartz M. Medication Preferences and Adherence Among Individuals With Severe Mental Illness and Psychiatric Advance Directives. Psychiatric Services. 2010;61(4):380-385.

(15) Srebnik D, Rutherford L, Peto T, Russo J, Zick E, Jaffe C y Holtzheimer P. The Content and Clinical Utility of Psychiatric Advance Directives. Psychiatric Services. 2005;56(5):592-498.

(16) Srebnik D y La Fond J. Advance Directives for Mental Health Treatment. Psychiatric Services. 1999;50(7)919-925.

(17) La Fond J y Srebnik D. The impact of mental Health advance directives on patient perceptions of coercion in civil commitment and treatment decisions. International Journal of Law and Psychiatry. 2002;25:537-555.

(18) Rittmannsberger H, Pachinger T, Keppelmüller P y Wancata J. Medication Adherence Among Psychotic Patients Before Admission to Inpatient Treatment. Psychiatric Services. 2004;55(2):174-179.

(19) Hamann J, Cohen R, Leucht S, Busch R y Kissling W. Do Patients with Schizophrenia Wish to Be Involved in Decisions About Their Medical Treatment? Am J Psychiatry. 2005;162:2382- 
ORIGINALES Y REVISIONES

2384.

(20) Ramos S. La toma de decisiones compartidas en pacientes con esquizofrenia: cuestiones médicas y éticas. Dilemata. 2012;10:263-277.

(21) Swanson J, Swartz M, Elbogen E, Van Dorn A, Wagner H, Moser L, Wilder Ch y Gilbert A. Psychiatric advance directives and reduction of coercive crisis interventions. J Ment Health. 2008;17(3):255-267.

(22) Tonelli M. Pulling the Plug on Living Wills. A Critical Analysis of Advance Directives. Chest. 1996;110:816-822.

(23) Buchanan A, y Brock D. Deciding for Others. The Ethics of Surrogate Decision Making. Cambridge: Cambridge University Press; 1989.

(24) Rich B. Personhood, Parenthood, and Clinical Practice. Reassessing Advance Directives. Psychology, Public Policy, and Law. 1998;4(3):610-628.

(25) Van Dorn R, Swartz M, Elbogen E, Swanson J, Kim M, Ferron J, McDaniel L, y Scheyett A. Clinicians'Attitudes Regarding Barriers to the Implementation of Psychiatric Advance Directives. Administration and Policy in Mental Health and Mental Health Services Research. 2006;33(4):449460 .

(26) Joshi K. Psychiatric Advance Directives. Journal of Psychiatric Practice. 2003;9:303306.

(27) Srebnik B, Appelbaum P y Russo J. Assessing Competence to Complete Psychiatric Advance With the Competence Assessment Tool for Psychiatric Advance Directives. Comprehensive Psychiatry. 2004;45(4):239-245.

(28) Sherman P. Computer-Assisted Creation of Psychiatric Advance Directives. Community Mental Health Journal. 1998;34(4):351-362. 